\section{CHALLENGES AHEAD}

'Lack of access to antiretroviral therapy is a global health emergency. To deliver antiretroviral treatment to the millions who need it, we must change the way we think and change the way we act.' Wise words spoken by Lee Jong-wook, Director General of the World Health Organisation. The 3 by 5 initiative was created by WHO because the six million people currently infected with HIV in the developing world need access to antiretroviral therapy to survive. Only 400000 people worldwide have this access. So WHO has set the targets at 3 million on treatment by 2005, and time is marching on. WHO has developed a strategic framework for this based on five pillars:

Global leadership, strong partnership and advocacy

- Urgent sustained country support

- Simple standardised tools for delivery

Effective reliable supply of medicines and diagnostics

- Rapidly identifying and reapplying new knowledge and successes.

We have just returned from an antiretroviral meeting in Dakar, Senegal, a vibrant interesting city where there is clear evidence of political leadership and support in the area of HIV. Health care professionals from West and Central Africa were keen to share experiences and discuss standardised strategies, and hungry for new knowledge and lessons learned. Very exciting developments are taking place in so many countries. Even in a country as battered as Zimbabwe we were told of a number of projects already enabling people to receive ART, and plans are in place for expansion.

Indeed, as we look back at more than a year of the Usapho Lwethu project in Gugulethu, Cape Town, I am so excited. It can be done - we can deliver treatment, people will take drugs faithfully, lives can be turned around and the progression of this horrible disease halted. The challenge for us all now is how to do this on a much greater scale. We need 53 sites in South Africa to be up and running in the next 6 months and 1.4 million HIVinfected people to be on treatment by 2007 to meet government targets.

There seems to be a hive of activity, but things will never move quickly enough, to which anyone who is HIV-infected and facing AIDS will bear testimony. There remains concern whether roll-out is happening at the same rate in all provinces - it is well known that some provinces have more to do than others, but there is no doubt that overall there is a huge amount of work to be done.

Pillar five is interesting and poses some challenges, since the field of HIV medicine is an incredibly fast moving one. No sooner is a paper or concept published than it is out of date. One needs to be on the conference circuit continuously to keep one step ahead of the most recent data.

Some interesting nevirapine data have recently come to light. A study published in the Journal of the Acquired Immune Deficiency Syndrome describes a higher rate of severe hepatotoxicity in non-HIV-infected than in HIV-infected individuals, and the rate in the latter group was higher with higher CD4 counts. The study therefore recommended that the use of post-exposure regimens containing nevirapine should be discouraged.

Montaner and colleagues ${ }^{2}$ have published findings that the risk of serious hepatotoxicity may be increased in individuals coinfected with hepatitis $B$ and $C_{1}$ abnormal liver enzymes at baseline or higher $\mathrm{CD} 4$ counts $(>350 / \mu l) .{ }^{2}$ A worrying paper from the Paris meeting by Lyons et al. ${ }^{3}$ entitled 'Nevirapine tolerability in HIV-infected women in pregnancy - a word of caution' indicates that there is a significant risk of nevirapineassociated hepatotoxicity in pregnant women, especially those with high CD4 cell counts, and that the progression to severe hepatoxicity may be explosive and not predicted by the patient's enzyme level at baseline. The risk of nevirapine-related hepatotoxicity and rash, which seems to be caused by an acute and idiosyncratic hypersensitivity reaction, increased with increasing CD4 cell counts. Hence women with CD4 counts > 250 and men with CD4 counts $>400$ or persons with recent exposure to HIV and therefore relatively normal immune responses are at particular risk. ${ }^{3}$

For these reasons, the Centers for Disease Control recommends against the use of nevirapine as post- exposure prophylaxis. ${ }^{4}$ While the 'once dosing' regimen used in South Africa for PMTCT is unlikely to cause hepatotoxicity, the data also suggest that the use of nevirapine in patients and pregnant women with high CD4 counts may be problematic and it may be necessary to measure CD4 counts before commencing a long-term nevirapine-containing regimen.

\section{LINDA-GAIL BEKKER \\ Managing Editor}

1. Serious adverse cutaneous an hepatic toxicities associated with nevirapine use by nonHIV-infected individuals. J Acquir Immune Defic Syndr 2004; 35(2): 120-125.

2. Montaner JS, Hall D, Carlier $\mathrm{H}$, et al. Analyses of four key clinical trials to assess the risk of hepatotoxicity with nevirapine: correlation with $\mathrm{CD} 4$ levels, hepatitis $\mathrm{B}$ and $\mathrm{C}$ seropositivity, and baseline liver function tests. Can J Infect Dis 2001; 12: suppl B, 31B.

3. Lyons F, Hopkins S, Mc Geary A, et al. Nevirapine tolerability in HIV-infected women in pregnancy - a word of caution. 2nd International AIDS Conference on HIV Pathogenesis and Treatment, Paris, 13-16 July 2003. Abstract LB27.

4. Serious adverse events attributed to nevirapine regimens for post exposure prophylaxis after HIV exposures worldwide, 1997-2000. MMWR 2001; 49: 1139-1156. 